\title{
ВЛИЯНИЕ НЕКОТОРЫХ ФАКТОРОВ НА УГОЛ РЕЗАНИЯ ВЕРТИКАЛЬНОГО НОЖА ПРИ РОТАЦИОННОЙ ОБРАБОТКЕ ПОЧВЫ
}

\author{
А.С. Григорян к.т.н., А.В. Алтунян к.т.н. \\ Национальный аграрный университет Армении \\ algrig1968@mail.ru, artur_altunyan@mail.ru
}

\section{СВЕДЕНИЯ}

\section{Ключевые слова:}

вертикальная фрреза,

угол установки,

крыло ножа,

диаметр фрезы,

угол резания

\begin{abstract}
АННОТАЦИЯ
При ротационной обработке почвы нож вертикальной фррезы постоянно находится в почвенном массиве. При жестком креплении ножей угол установки ножа выбирается с таким расчетом, чтобы в самой нагруженной части угол резания находился в оптимальном интервале значений 20-30․ При этом в остальных частях траектории, особенно за фронтальным участком, угол резания достигает недопустимых значений, и происходит сгребание уже обработанной почвы внутренним крылом ножа.

В статье исследовано теоретическое влияние отдельных факторов на величину угла резания ножа: ширина крыла ножа, угол заострения, кинематический параметр и диаметр фррезы.
\end{abstract}

\section{Введение}

В настоящее время для обработки почвы широкое применение нашли машины с ротационными почвообрабатывающими рабочими органами. Этому в большей мере способствует тот фракт, что почвообрабатывающие фрезы наиболее полно соответствуют агротехнологическим требованиям по качеству крошения почвы и уничтожению сорной растительности. Однако надо заметить, что по энергетическим затратам они гораздо превосходят машины с пассивными рабочими органами.

Как указывают авторы (Г.Н. Синеоков, И.М. Панов, 1977), углы резания ротационных ножей на отдельных траекториях движения значительно превосходят углы резания обычных рабочих органов более чем в два раза (у плужных лемехов, культиваторных лап он равен 20300). Этим фрактом в значительной мере объясняется повышенный расход энергии на их работу. В то же время надо также отметить, что изменчивость угла резания у ротационных ножей в весьма обширных интервалах не только повышает энергоемкость технологического процесса, но и отрицательно влияет на технологическое качество обработки почвы.

В статье рассмотрено влияние отдельных фракторов (ширина крыла и угол заострения ножа, диаметр фрезы, кинематический параметр ротационной машины) на изменение величины угла резания и на технологическое качество процесса обработки почвы.

\section{Материалы и методы}

Изучением влияния угла резания на энергоемкость и качество технологического процесса при ротационной обработке почвы занимались многие исследователи: Г. Попов, П. Павлов, А. Далин, В. Зоне, И. Панов, Ф. Канарев, Ю. Матяшин и другие. Эксперименталь- 
ными данными, полученными вышеперечисленными авторами, в основном подтверждается тот подход, при котором потребная мощность фрезерования с увеличением угла резания нарастает.

Так, по экспериментальным данным П. Павлова (П.В. Павлов, 1952), при изменении угла резания с $17^{0}$ до $30^{\circ}$ происходит относительно медленный рост потребной мощности, а начиная с $30^{\circ}$ до $42^{\circ}$ величина мощности резко возрастает. При угле резания $42^{\circ}$ мощность фррезерования на 60-80 \% больше, чем при $\beta=30^{\circ}$, и вдвое больше, чем при $\beta=17^{\circ}$. В результате аналогичных опытов с Г-образными ножами с углами резания $15 ; 19 ; 25 ; 30^{\circ}$ и одинаковым углом заточки 150, проведенных в почвенном канале, В. Зоне (W. Söhne, 1957) приходит к выводу, что допустимым является угол резания в пределах 20-250. Примерно такие же зависимости были получены в опытах А. Далина (А.Д. Далин, П.В. Павлов, 1950) и Ю. Матяшина (Ю.И. Матяшин, 1988), на основе которых они приходят к выводу, что оптимальным является угол резания в интервалах $25-35^{\circ}$. В работе (Ф.М. Канарев, 1983) исследовалась зависимость энергоемкости фрезерования от угла установки ножей во влажных почвах, отведенных под рис. Наименьшие показатели затраты энергии получены при отрицательных значениях заднего угла резания 3-50.

Обобщая и анализируя перечисленные данные, И. Панов (Г.Н. Синеоков, И.М. Панов, 1977) считает, что оптимальной величиной угла резания для почвообрабатывающих фрез является 20-30․

Однако надо отметить, что эти исследования проводились для фрез с горизонтальной осью вращения, у которых технологический процесс имеет прерывистый характер. А так как угол резания ножа непрерывно изменяется по мере вращения барабана, то некоторая часть этих изменений приходится на ту часть вращения барабана, когда нож фррезы уже находится вне почвенного массива, тем самым не оказывая существенного влияния на энергоемкость технологического процесса. Также допускается, что активный процесс резания приходится на еще не обработанную почвенную зону, после чего нож перемещается в уже разхрыленную зону, где энергозатраты несравнимо малы.

Иначе проходит технологический процесс у фрез с вертикальной осью вращения. Здесь в течение всего процесса ножи находятся в почвенном массиве, и интервал изменений угла резания в этом случае оказывает существенное влияние на энергозатраты и качество технологического процесса.

На угол резания ножа влияют многочисленные кинематические и конструкционные факторы техно- логического процесса: кинематический параметр ротационной обработки - $\lambda=V_{\circ} / V_{n}$, где $V_{n}$ - поступательная, а $V_{\text {o }}$ - окружная скорость ножа; радиус вращения - $R$, ширина крыла - $L$, угол заострения - $i$, угол установки - $\gamma$.

Для определения угла установки ножа с учетом ширины крыла в работе (3) предлагается зависимость (рис. 1):

$$
\gamma=\arccos \left(\frac{1}{\lambda}+\frac{L}{2 R}\right) .
$$

Автор исходит из того, что самым нагруженным участком резания является точка пересечения $A$ траектории движения ножа с прямой, проходящей через мгновенный центр вращения (рис. 1).

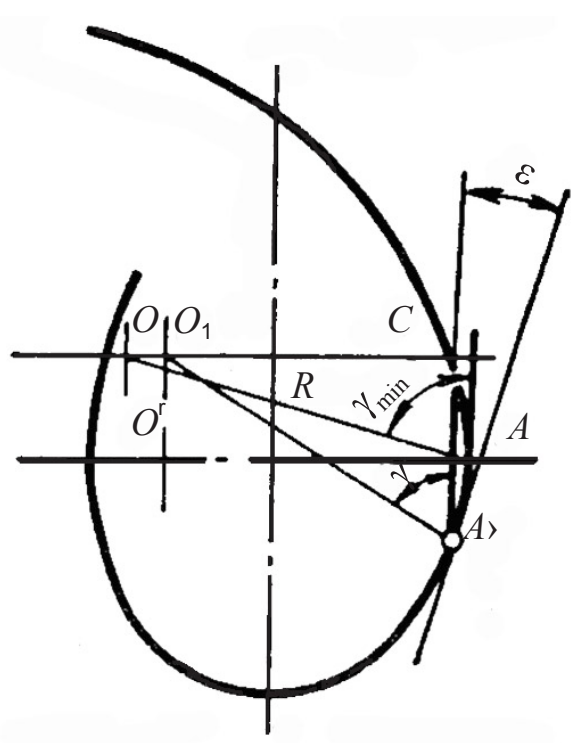

Рис. 1. Схема для определения угла установки ножа с учетом ширины крыла ножа (Г.Н. Синеоков, И.М. Панов, 1977).

При таком подходе к вопросу об оптимальном угле установки ножа действительно обеспечивается минимальный угол резания в самой нагруженной точке траектории резания. Однако при расчете по рис. 1 угол установки ножа в противоположной от точки $A$ стороне не обеспечивает оптимального угла резания, так как он получается слишком завышенным. Ввиду этого нарушается условие скользящего движения почвы относительно внутренней поверхности крыла ножа, вследствие чего почва сгребается внутренним крылом ножа и выносится из рабочей зоны фрезы, что приводит к оголению почвы. 


\section{Результаты и анализ}

Для выявления основных соотношений между углами вертикального ножа воспользуемся схемой на рис. 2. Из схемы видно, что положение ножа в отдельных участках траектории непрерывно меняется, чем обусловлено также изменение угла резания. Ввиду того что при жестком креплении ножей на диске фрезы вышеописанное явление неизбежно, то надо хотя бы стремиться к тому, чтобы диапазон изменений угла резания не сильно варьировался при одном обороте ножа. Для соблюдения данного условия при проектировании машин по возможности необходимо выбрать соотвествующие конструктивные параметры и технологический режим работы.

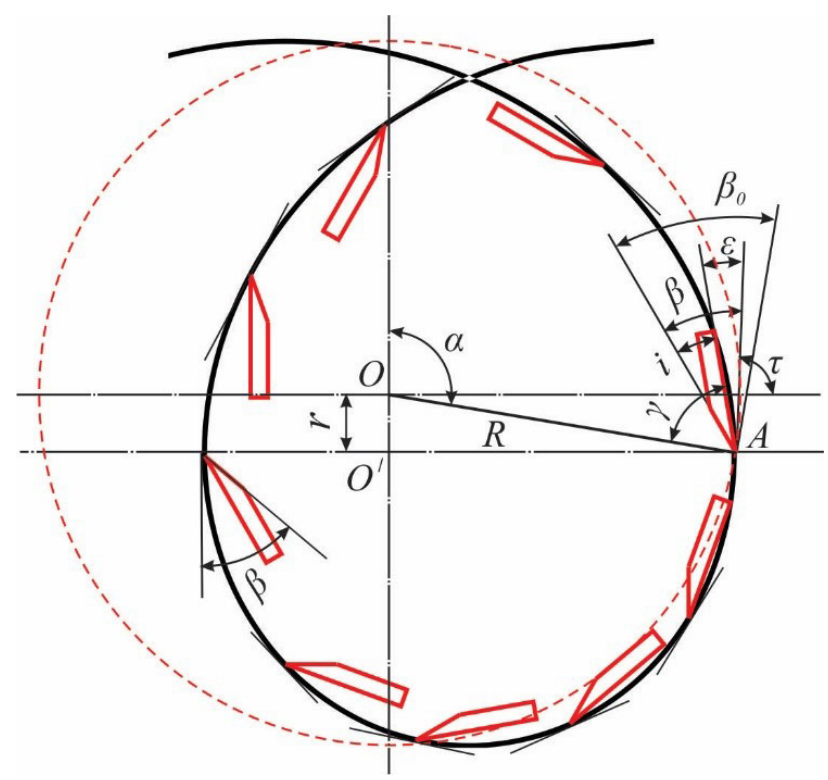

Рис. 2. Определение углов установки и резания ножа вертикальной фрезы (составлен авторами).

Для определения угла резания ножа воспользуемся схемой (рис. 2). Из схемы видно, что

$$
\beta=270-\gamma+i-\tau-\alpha
$$

где $\gamma$ - угол установки ножа, $i$ - угол заострения, $\alpha$ - угол поворота ножа, $\tau$ - угол наклона касательной к циклоиде относительно оси абсцисс (3):

$$
\tau=-\operatorname{arctg}\left(\frac{\lambda \sin a}{1+\cos a}\right)
$$

На рис. 3 и 4 показаны графики диапазона изменений угла резания вертикального ножа при одном обороте фрезы по условию определения угла установки по формуле (1) при различных значениях кинематического параметра ротационной машины.

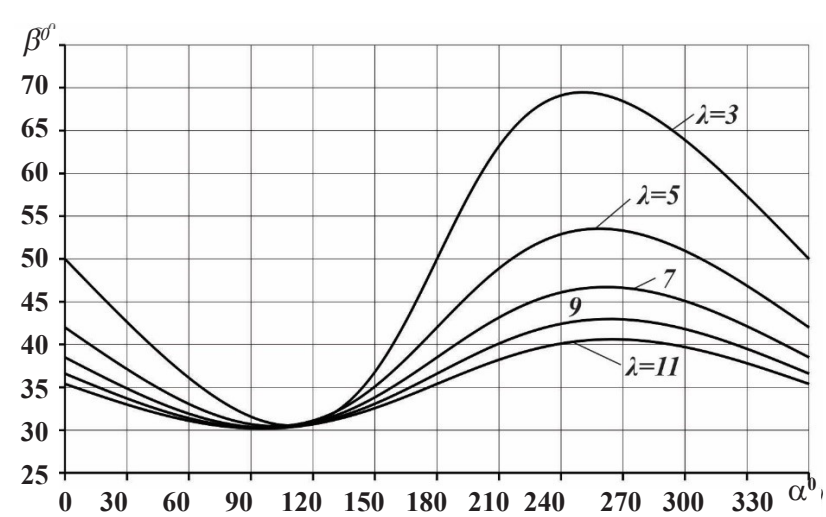

Рис. 3. Изменение угла резания вертикального ножа от угла поворота при разных кинематических параметрах ротационной машины $\left(R=20 \mathrm{~cm}, L=7 \mathrm{cM}, \quad i=20^{\circ}\right)$ (составлен авторами).

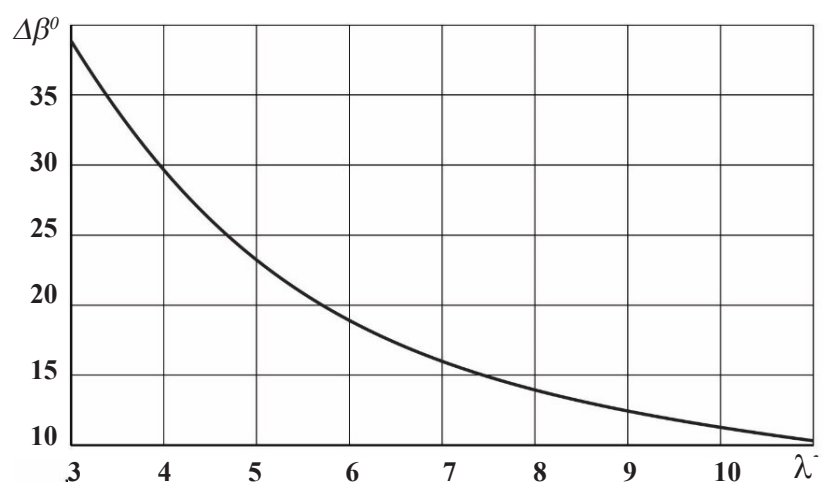

Рис. 4. Диапазон изменения углов резания от кинематического параметра ротационной машины $(R=20 \mathrm{~cm}, L=7$ cм, $i=20^{\circ}$ ) (составлен авторами).

Из приведенных графиков видно, что разница значений углов резания, при неизменности геометрических и технологических параметров, значительно выше при низких значениях кинематического параметра (до 8-9). Дальнейшее увеличение кинематического параметра не оказывает существенного влияния на характер и величину этих изменений. Поэтому при выборе режима работы ротационной машины нецелесообразно выбирать кинематический параметр ниже 8-9. Однако даже при больших значениях кинематического параметра, конкретно в приведенном режиме работы, не удается обеспечивать такое значение угла резания, при котором он находился бы в оптимальных интервалах в 20-30. 
Некоторого снижения значения угла резания можно достичь снижением угла заточки лезвия ножа и заднего угла резания, но ресурс первого способа ограничен, так как чрезмерное снижение угла заострения приводит к быстрому затуплению и выкрашиванию лезвия ножа. Возможности снижения заднего угла резания препятствует вертикальная стенка борозды.

На угол резания влияет также ширина крыла ножа. Возможность снижения заднего угла резания обусловлена уменьшением ширины крыла ножа $L$ (рис. 2), так как при этом можно выбрать максимально возможный угол установки ножа $\gamma$. На рис. 5 приведена зависимость изменения угла установки ножа от соотношений при разных значениях кинематического режима ротационной машины.

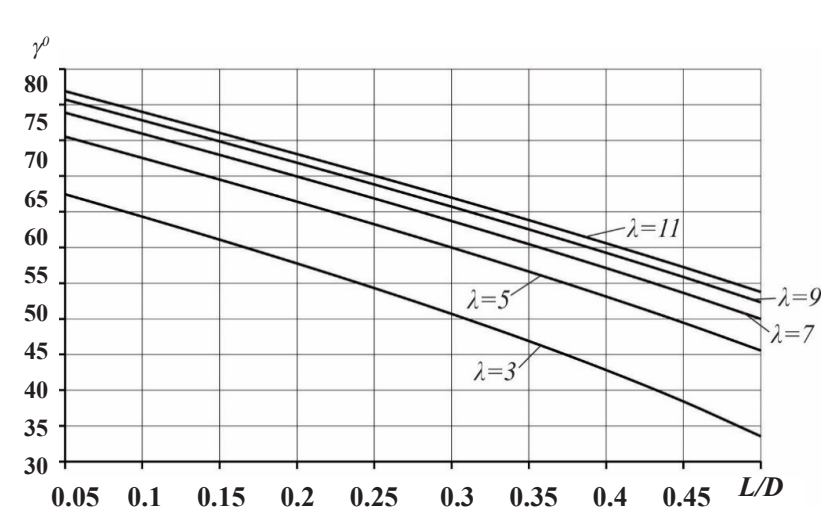

Рис. 5. Зависимость угла установки ножа ү от соотношений L/D (составлен авторами).

Из графика видно (рис. 5), что для вертикальной фррезы оптимальными являются значения параметров $L D=0,1 \ldots 0,15 ; \lambda \geq 9$, при которых можно считать, что угол резания ножа находится в интервале допустимых значений.

\section{Заключение}

В зависимости от выбранных конструктивных и кинематических параметров ротационной машины с вертикальной осью вращения диапазон изменений угла резания порой может достигать недопустимых величин, при которых в заднем секторе вращения может происходить сгребание внутренним крылом ножа уже обработанной почвы.

Негативное влияние этих изменений можно снизить путем выбора соответствующих конструктивных и кинематических параметров ротационной машины.

В результате теоретических исследований и анализа полученных зависимостей оптимальным можно считать значения следующих параметров ротационной фрезы:

$$
L D=0.1 \ldots 0.15 ; \lambda \geq 9 ; i=15 \ldots 20^{\circ} .
$$

Данные теоретического исследования послужат предпосылкой для разработки и проектирования принципиально нового рабочего органа фрезы с вертикальной осью вращения, ножи которого будут способны авторегулироваться в течении всего процесса резания, тем самым обеспечивая постоянный угол резания.

\section{Литература}

1. Далин А.Д., Павлов П.В. Ротационные грунтообрабатывающие и землеройные машины. - М.: Машгиз, 1950. - 258 c.

2. Канарев Ф.М. Ротационные почвообрабатывающие машины и орудия. - М.: Машиностроение, 1983. $-142 \mathrm{c}$.

3. Матяшин Ю.И. Расчет и проектирование ротационных почвообрабатывающих машин. - М.: Агропромиздат, 1988. - 174 с.

4. Павлов П.В. Исследование сил в почвенных фрезах. - М.: Сельхозгиз, 1952.

5. Попов Г.Ф. К методике проектирования рабочих органов фрезерных культиваторов. - М.: ВИСХОМ, 1973. - 497 c.

6. Синеоков Г.Н., Панов И.М. Теория и расчет почвообрабатывающих машин. - М.: Машиностроение, 1977. - 322 с.

7. Söhne W. (1957). Einfluss von Form und Anordnung der Werkzeuge auf Antriebsmomönte von Ackerfräsen. Grundl. d. Landtechn, - N. 9, - pp. 696-787. 


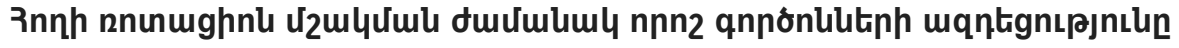

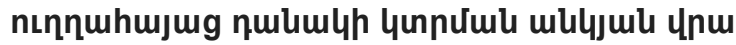

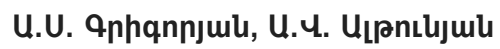

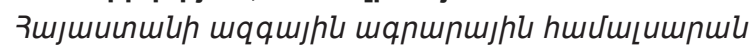

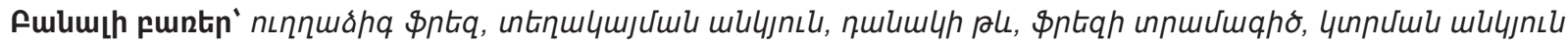

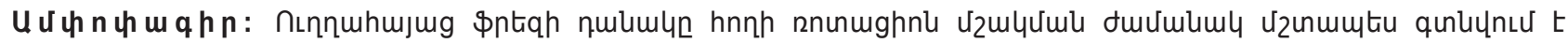

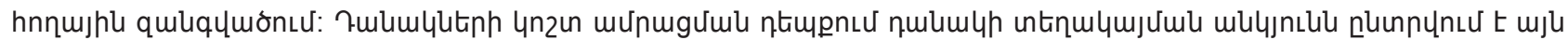

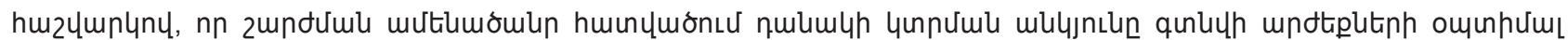

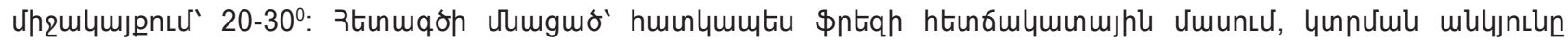

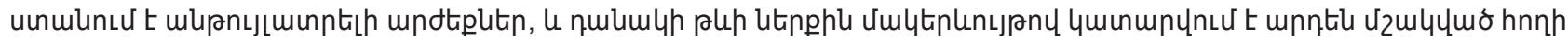
ptinmpuuhnul:

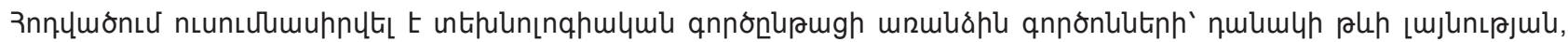

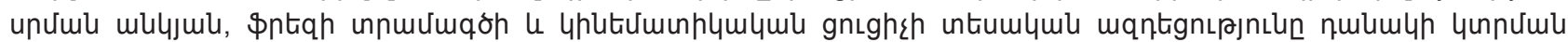
mulıرul unu:

\section{The Effect of Some Factors on the Cutting Angle of the Vertical Blade during Rotary Soil Tillage}

\section{A.S. Grigoryan, A.V. Altunyan}

Armenian National Agrarian University

Keywords: vertical cutter, setting angle, blade wing, cutter diameter, cutting angle

Abstract. During the rotary soil tillage the blade of vertical cutter is constantly in the soil mass. In case of rigid attachment of the blades, the setting angle is selected so that in the most loaded part the cutting angle is in the optimal value range of $20-30^{\circ}$. At the same time, in the remaining parts of the trajectory, especially behind the frontal section, the cutting angle reaches unacceptable values and the already cultivated soil is raked with the inner blade of the knife.

The article considers the theoretical impact of individual factors, such as blade width, cutting-point angle, kinematic parameters and cutter diameter on the value of the blade cutting angle.

Статья опубликована в рамках научной темы 18Ap_2k13 комитета Науки МОН PA. 